\title{
Generation of Triplet Excited States via Photoinduced Electron Transfer in meso-anthra-BODIPY: Fluorogenic Response toward Singlet Oxygen in Solution and in Vitro
}

Mikhail A. Filatov, ${ }^{*}{ }^{\dagger}$ ○ Safakath Karuthedath, ${ }^{\ddagger}$ Pavel M. Polestshuk, ${ }^{\S}$ Huguette Savoie, ${ }^{\text {I Keith J. Flanagan, }}{ }^{\dagger}$ Cindy Sy, ${ }^{\dagger}$ Elisabeth Sitte, ${ }^{\dagger}$ Maxime Telitchko, ${ }^{\dagger}$ Frédéric Laquai, ${ }^{\ddagger}{ }^{\ddagger}$ Ross W. Boyle, ${ }^{*}, \|$ and Mathias O. Senge ${ }^{*}+$ (0)

${ }^{\dagger}$ School of Chemistry, SFI Tetrapyrrole Laboratory, Trinity Biomedical Science Institute, Trinity College Dublin, The University of Dublin, 152-160 Pearse Street, Dublin 2, Ireland

${ }^{\ddagger}$ King Abdullah University of Science and Technology (KAUST), KAUST Solar Center (KSC), Physical Sciences and Engineering Division (PSE), Material Science and Engineering Program (MSE), Thuwal 23955-6900, Saudi Arabia

${ }^{\S}$ Department of Chemistry, M.V. Lomonosov Moscow State University, Leninskie Gory, 1/3 Moscow 119991, Russia

"Department of Chemistry, University of Hull, Cottingham Road, Kingston-upon-Hull HU6 7RX, United Kingdom

Supporting Information

ABSTRACT: Heavy atom-free BODIPY-anthracene dyads (BADs) generate locally excited triplet states by way of photoinduced electron transfer (PeT), followed by recombination of the resulting charge-separated states (CSS). Subsequent quenching of the triplet states by molecular oxygen produces singlet oxygen $\left({ }^{1} \mathrm{O} 2\right)$, which reacts with the anthracene moiety yielding highly fluorescent species. The steric demand of the alkyl substituents in the BODIPY subunit defines the site of ${ }^{1} \mathrm{O}_{2}$ addition. Novel bis- and tetraepoxides and bicyclic acetal products, arising from rearrangements of anthracene endoperoxides were isolated and characterized. ${ }^{1} \mathrm{O}_{2}$ generation by $\mathrm{BADs}$ in living cells enables visualization of the dyads distribution, promising new imaging applications.

$\mathrm{O}$ ptical probes based on photoinduced electron transfer (PeT) in donor-acceptor dyads have broad use in diagnostics, particularly for detection of biomolecules, metal ions, reactive oxygen species (ROS) and measurement of intracellular $\mathrm{pH} .{ }^{1}$ The PeT process leads to formation of nonemissive charge-separated states (CSS) that decay back to the ground state via different pathways. Among those is recombination of CSS, which may lead to locally excited triplet states of the molecule. ${ }^{2}$ Recently this process has attracted attention as a method to increase intersystem crossing without relying on the heavy atom effect. ${ }^{3}$

The possibility of singlet oxygen $\left({ }^{1} \mathrm{O}_{2}\right)$ generation by donoracceptor dyads has not been realized so far in a practical sense. It could be expected that ${ }^{1} \mathrm{O}_{2}$ generation by PeT-based optical probes in biological environments would affect their optical response and simultaneously induce cytotoxicity. This is of concern especially in the case of ROS detection, where sensitization of ${ }^{1} \mathrm{O}_{2}$ by the probe itself may lead to false positives and incorrect interpretations. ${ }^{4}$ On the other hand, PeT-mediated ${ }^{1} \mathrm{O}_{2}$ generation could provide a new tool for theranostic applications, because the process of charge separation can be turned on/off by various stimuli. Herein we report readily accessible heavy atom-free BODIPY-anthracene dyads (BADs) that can act as efficient triplet sensitizers, and become fluorescent in response to the generated ${ }^{1} \mathrm{O}_{2}$.

Although a number of triplet sensitizers based on halogenated BODIPYs have been reported in the past decade, ${ }^{5}$ observations of triplet excited states formation in heavy atom-free BODIPYs are rare. ${ }^{6}$ In our search for efficient donor-acceptor photosensitizers, we focused on BADs 1 and 2 (Scheme 1). BODIPYs

Scheme 1. Photoinduced Transformations of BADs

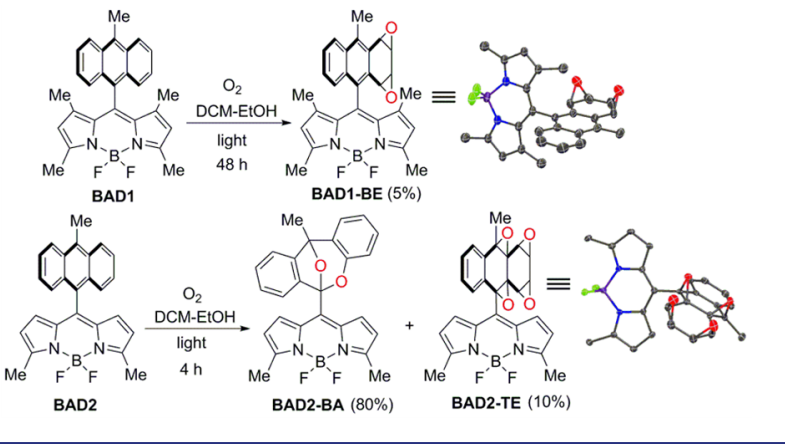

are known to be efficient energy and electron acceptors when combined with anthracene. ${ }^{7}$ Although compound BAD1 has been reported to exhibit PeT, no triplet excited states formation has been noted. ${ }^{8}$

Upon broad-band visible light irradiation of air-saturated solutions of BAD1 in a range of polar solvents we observed, to our surprise, completely selective formation of BAD1-BE, which could be isolated in 5\% yield along with recovered unreacted starting material (Scheme 1). In contrast, irradiation of BAD2 under the same conditions resulted in complete conversion of

Received: January 17, 2017

Published: April 13, 2017 
the substrate and formation of two products, bicyclic acetal derivative (BAD2-BA) and tetraepoxide (BAD2-TE), which were isolated in $80 \%$ and $10 \%$ yields, respectively. The structures of the products were confirmed by NMR spectroscopy and X-ray crystallography (for details, see Supporting Information (SI)). Unlike BADs 1 and 2, isolated compounds exhibit bright fluorescence independent of the solvent polarity. For instance, the emission quantum yields of BAD 1-BE in $\mathrm{CH}_{2} \mathrm{Cl}_{2}$ and hexane were determined to be 0.91 and 0.89 , respectively.

The formation of these products appears to be due to the sensitization of oxygen and subsequent $[4+2]$ cycloaddition of the resulting ${ }^{1} \mathrm{O}_{2}$, which is typical for anthracene derivatives. ${ }^{9}$ Singlet oxygen quantum yields were measured using 1,3diphenylisobenzofuran as ${ }^{1} \mathrm{O}_{2}$ trap, giving values of 0.67 and 0.38 in ethanol, for $\mathrm{BAD1}$ and $\mathrm{BAD2}$, respectively. To understand the mechanism of ${ }^{1} \mathrm{O}_{2}$ formation, we studied the excited state dynamics of the dyad BAD1 by broad-band vis-NIR subpico- to microsecond transient absorption (TA) pumpprobe spectroscopy.

Immediately after photoexcitation with fs pulses at $355 \mathrm{~nm}$, a broad band around $360 \mathrm{~nm}$ due to the anthracene's singlet excited state $\left(S_{1}\right)$ absorption, which partially overlaps with the ground state photo bleach (PB), was observed (Figure 1a). The
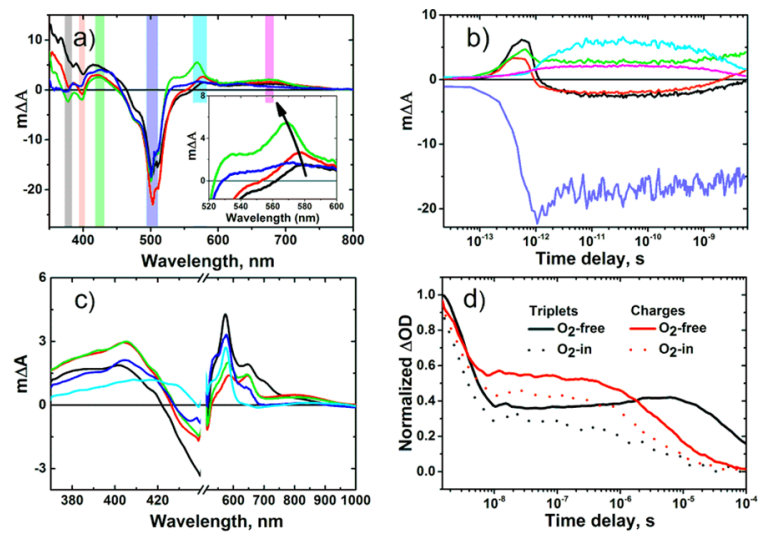

Figure 1. (a) ps-ns Transient absorption spectra of BAD1 in dimethylformamide upon excitation at $355 \mathrm{~nm}$ with $35 \mathrm{fs}$ pulses at delay times of $600 \mathrm{fs}$ (black line), $1 \mathrm{ps}$ (red line), $100 \mathrm{ps}$ (green line), and $5 \mathrm{~ns}$ (blue line). The inset shows the blue shift of the TA spectra to 570 $\mathrm{nm}$. (b) Kinetics monitored at $380 \mathrm{~nm}$ (black line), $400 \mathrm{~nm}$ (red line), $425 \mathrm{~nm}$ (green line), $505 \mathrm{~nm}$ (violet line), $570 \mathrm{~nm}$ (cyan line), and 680 $\mathrm{nm}$ (magenta line) as indicated by vertical colored bars. (c) ns $-\mu \mathrm{s}$ Transient absorption spectra of degassed BAD1 solutions following excitation at $355 \mathrm{~nm}$ by $700 \mathrm{ps}$ laser pulses. The spectra were integrated from 3 to $5 \mathrm{~ns}$ (black line), $10-100 \mathrm{~ns}$ (red line), $0.1-1 \mu \mathrm{s}$ (green line), $1-5 \mu$ s (blue line), and 10-100 $\mu$ s (cyan line). (d) Kinetics observed for the bands at 570 and $680 \mathrm{~nm}$, assigned to the BODIPY triplet state and anthracene radical-cation, respectively, in the absence and presence of oxygen (solid and dotted lines, respectively).

concomitant decay of this band and simultaneous rise of the bleach at $505 \mathrm{~nm}$ indicate ultrafast energy transfer (EnT) from anthracene to BODIPY subunit, populating the singlet state of the latter, $S^{\mathrm{BDP}}$. Furthermore, another absorption band grows at $580 \mathrm{~nm}$, and it was assigned to the BODIPY radical-anion $\left(\mathrm{BDP}^{-\bullet}\right),{ }^{10}$ forming due to the PeT process. This band rises during the first $100 \mathrm{ps}$, simultaneously shifting to $570 \mathrm{~nm}$, indicating a transition of the radical-anion to another excited state (see inset of Figure 1a). Synchronously with the rise of the $\mathrm{BDP}^{-} \bullet$ absorption $(580 \mathrm{~nm})$, yet another absorption band, centered at $680 \mathrm{~nm}$ rises, presumably due to the anthracene radical-cation $\left(\mathrm{Ant}^{+\bullet}\right)$, in line with previous reports. ${ }^{11}$ Fitting of the $\mathrm{PB}$ decays at 380 and $400 \mathrm{~nm}$ and the rise of $\mathrm{BDP}^{-\bullet}$ and Ant $^{+\bullet}$ bands (Figure $1 \mathrm{~b}$ ) yields time constants of 1.15 and 0.54 ps for the EnT and PeT processes, respectively.

In the ns $-\mu$ s TA experiments, a rise of an absorption band at $570 \mathrm{~nm}$ over $1 \mu \mathrm{s}$ was observed, indicating formation of longlived states (Figure 1c). Previous reports on the TA spectra of BODIPY support the assignment of this band to the BODIPY triplet state $\left(\mathrm{T}^{\mathrm{BDP}}\right)$ absorption. ${ }^{10 \mathrm{~b}}$ The band at $570 \mathrm{~nm}$ was quenched and decayed faster in the presence of oxygen (Figure $1 d)$. In contrast, the anthracene radical cation-absorption band at $680 \mathrm{~nm}$ was impacted by oxygen significantly less. The $\mathrm{T}^{\mathrm{BDP}}$ lifetime in the absence of $\mathrm{O}_{2}$ was determined to be $41 \mu \mathrm{s}$. The observed transition from the bands originating in CSS to the absorption by the triplet suggests that the formation of CSS is a prerequisite for populating of $\mathrm{T}^{\mathrm{BDP}}$.

The frontier molecular orbitals diagram (Figure 2a) shows that the two highest occupied orbitals $\pi_{\text {ant }}$ and $\pi_{\mathrm{BDP}}$ located on the

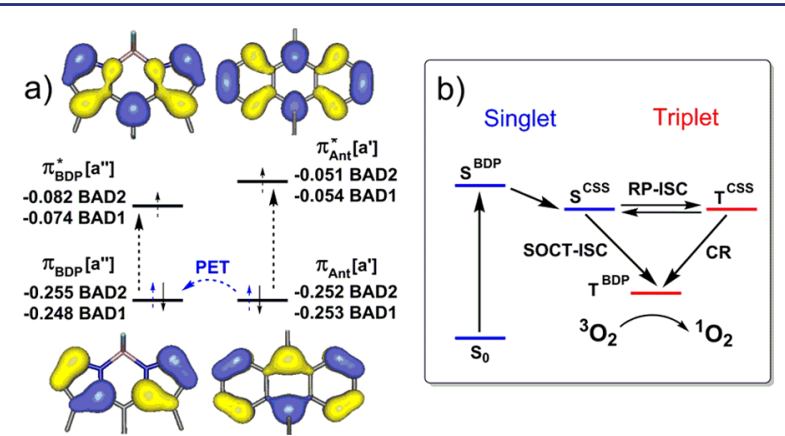

Figure 2. (a) Frontier molecular orbitals and their energies (in a.u.) for BADs 1 and 2. (b) Diagram demonstrating transitions between excited states in BAD1.

anthracene and BODIPY subunit, respectively, are nearly degenerate. Density functional theory (DFT) calculations (see SI for computational details) confirm that in BAD1 PeT could take place from $\pi_{\text {ant }}$ to singly occupied $\pi_{\mathrm{BDP}}$, leading to singlet charge transfer state $S^{\mathrm{CSS}}$ that is $0.4 \mathrm{eV}$ more stable than the $\mathrm{S}^{\mathrm{BDP}}$ excited state. Unlike the valence excited states, CSS has very low ferromagnetic exchange coupling integral due to negligible overlap of singly occupied orbitals $\pi_{\text {ant }}$ and $\pi^{*}{ }_{\text {BDP }}$ located in mutually orthogonal molecular moieties, thus leading to a very small singlet-triplet energy gap (S-T gap). Two pathways for triplet state generation from CSS may yield the lowest local $\mathrm{T}^{\mathrm{BDP}}$ state (Figure 2b): spin-orbit charge transfer intersystem crossing (SOCT-ISC) and radical pair intersystem crossing (RP-ISC)), followed by triplet charge recombination. ${ }^{12}$ As shown in extensive works of Wasielewski and co-workers, ${ }^{13}$ SOCT-ISC prevails for systems with strong electronic couplings, requiring short distances between the subunits (4.3 $\AA$ in BAD1). On the other hand, due to the small S-T gap in the RP state, mixing of $S^{\mathrm{CSS}}$ and $\mathrm{T}^{\mathrm{CSS}}$ states is possible due to, e.g., electronnuclear hyperfine coupling. More detailed studies are necessary to distinguish between mechanisms governing spin interconversion in BADs.

The observed PeT process is clearly manifested in the spectroscopic properties of BADs. The fluorescence of the BODIPY is quenched in polar solvents as evidenced by the negligible values of $\Phi_{\mathrm{f}}$, compared to the strong emission in nonpolar solvents (Table S3). A broad emission band at $610 \mathrm{~nm}$ 
was observed in polar solvents. Such red-shifted broad emission bands arising from charge transfer excited states were reported for various donor-acceptor systems. ${ }^{14}$ DFT calculations in vacuo show that $S^{C S S}$ state is approximately $0.2 \mathrm{eV}$ higher in energy than the valence $S^{\mathrm{BDP}}$ state. The dipole moment for the $S^{\mathrm{CSS}}$ state was computed to be $\mu=19 \mathrm{D}$ in vacuo, much higher than that for the valence $S^{\mathrm{BDP}}$ state $(5 \mathrm{D})$. Interactions of CSS with polar solvent result in a decrease of the $S^{\text {CSS }}$ state dipole moment to $1.1 \mathrm{D}$ and change the relative energy ordering of the $S^{\mathrm{BDP}}$ and $S^{\mathrm{CSS}}$ states, making PeT process favorable.

When irradiated with monochromatic or broad-band visible light, air-saturated solutions of BADs in polar solvents showed a gradual increase in fluorescence (Figure $3 b$ ). For instance,
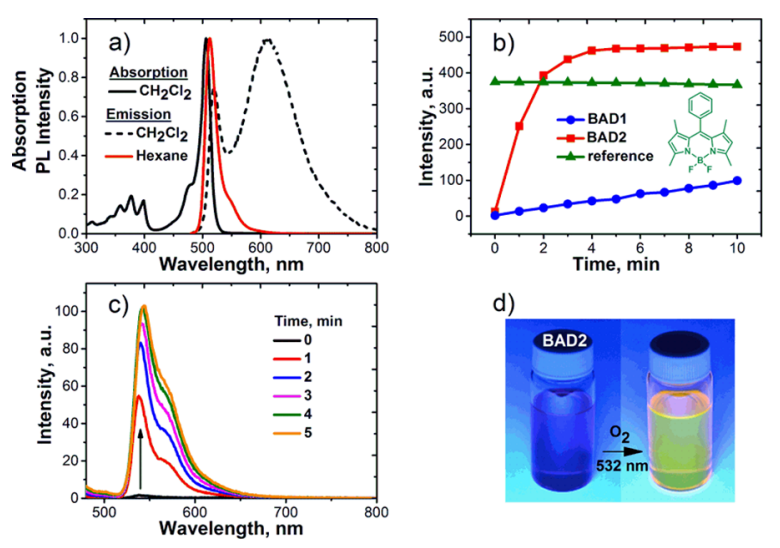

Figure 3. (a) Absorption and emission spectra of BAD1. (b) Changes of the emission intensity upon irradiation of BADs and reference compound solutions in $\mathrm{CH}_{2} \mathrm{Cl}_{2}\left(5 \times 10^{-5} \mathrm{M}\right)$ with broad-band visible light. (c) Change of BAD2 emission upon irradiation with $532 \mathrm{~nm}$ laser $\left(10 \mathrm{~mW} \mathrm{~cm}^{-2}\right)$. (d) Photo of BAD2 solution before and after $5 \mathrm{~min}$ of irradiation, taken under excitation with $365 \mathrm{~nm}$ light.

irradiation of BAD2 solution results in up to 100-fold increase of fluorescence intensity due to formation of compounds BAD2BA and BAD2-TE. No change in the emission was observed upon irradiation of the solutions in hexane even for longer periods of time (Figure S6), confirming that the dyads do not generate ${ }^{1} \mathrm{O}_{2}$ in the absence of the PeT process.

The formation of bicyclic acetal and tetraepoxide products from BAD2 is likely to take place via a 9,10-endoperoxide intermediate (Scheme 2). The rearrangement of endoperoxides

Scheme 2. Tentative Mechanism of the Formation of Fluorescent Products

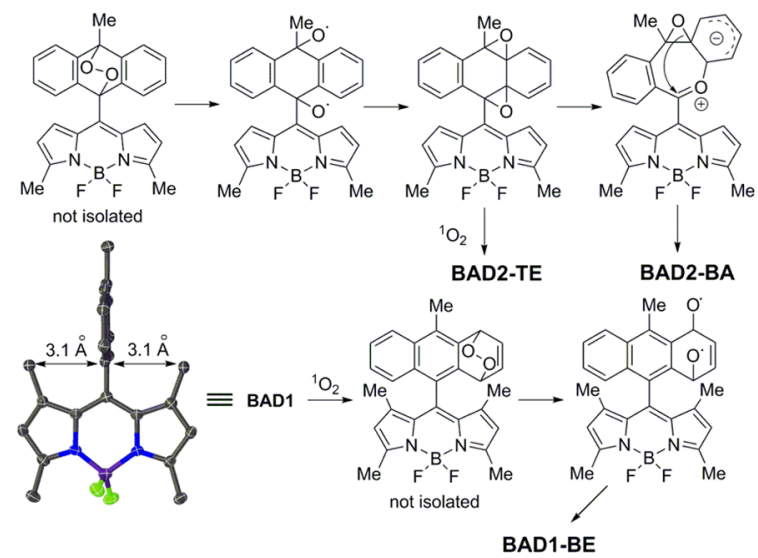

into bisepoxides can be induced either thermally or photochemically. ${ }^{9}$ The process is caused by homolytic cleavage of the peroxide $\mathrm{O}-\mathrm{O}$ bond, followed by rearrangement to more stable bisepoxides. Commonly, such bisepoxides containing a cyclohexadiene ring could not be isolated but only trapped with dienophiles. ${ }^{15}$ Indeed, we found no traces of this intermediate in the reaction mixture. According to previous reports, the formation of a bicyclic acetal from bisepoxide may take place via heterolytic cleavage of the epoxide $\mathrm{C}-\mathrm{C}$ bond, leading to an ylide-type bipolar intermediate. ${ }^{15 \mathrm{~b}}$ This is followed by $\mathrm{C}-\mathrm{O}$ bond rupture of a second epoxide fragment, leading to rearomatization of the lateral ring and formation of the acetal bridge. The rearrangement competes with addition of ${ }^{1} \mathrm{O}_{2}$ molecule to the diene moiety leading to BAD2-TE.

In contrast, bisepoxide BAD1-BE is stable and showed no formation of the rearrangement products. Its formation likely proceeds via the mechanism discussed above, involving $\mathrm{O}-\mathrm{O}$ homolytic cleavage and subsequent isomerization. The addition of ${ }^{1} \mathrm{O}_{2}$ to the outer ring in this case is surprising, as the central 9,10 -site is the most reactive, based on frontier molecular orbital analysis. The influence of steric factors on the regioselectivity of endoperoxide formation has been reported for acenes with bulky substituents at the ortho positions of the aryl groups. ${ }^{16}$ Comparison with BAD2 shows that the unusual reactivity of BAD1 can be attributed to the effect of methyl substituents in position 4 of the BODIPY core. This can be seen in the XRD data, where C-4 methyl substituents in BAD1 are forming a steric-like shield of the C-9 position of the anthracene unit. Introduction of methyl groups into the BODIPY pyrrole rings shields the inner ring of the orthogonal anthracene residue, making the approach of ${ }^{1} \mathrm{O}_{2}$ molecule difficult. Different reactivity of BADs toward ${ }^{1} \mathrm{O}_{2}$ accounts for the variations in their fluorescence response (Figure $3 \mathrm{~b}$ ) due to the cycloaddition to the anthracene moiety, which takes place considerably faster for BAD2.

The rise of BAD2 fluorescence due to cycloaddition reaction is manifested even at $1 \mu \mathrm{M}$ concentration, and it reaches intensities comparable to those of emission of a strongly fluorescent reference BODIPY compound (Figure S7). It was of special interest to investigate whether the sensitization process can be reproduced in live cells. For this purpose, we generated appropriate water-soluble derivatives (Scheme 3). Substitution

\section{Scheme 3. Synthesis of Water-Soluble BADs Derivatives}

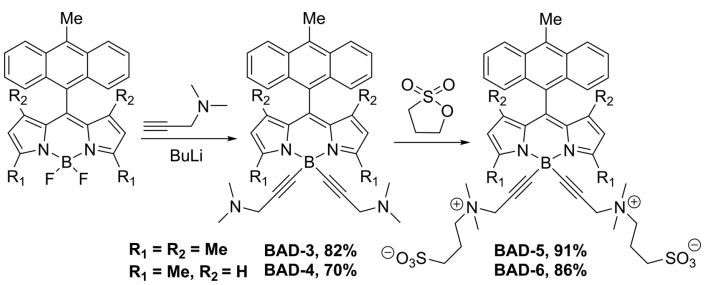

of fluorine atoms with $\mathrm{N}, \mathrm{N}$-dimethylaminopropyne- 1 residues gave corresponding BADs 3 and 4. Quaternization of the dimethylamino group with 1,3-propane sultone then gave BADs 5 and 6 , bearing zwitterionic fragments (betaine) that imparted the desired aqueous solubility.

To examine the fluorescence response of BADs 5 and 6 toward self-sensitized ${ }^{1} \mathrm{O}_{2}$ in cells, human breast cancer (MDAMB-468) cells were incubated with BADs 5 and $6(1 \mu \mathrm{M})$ followed by irradiation with broad-band visible light (400-700 $\mathrm{nm}, 23.8 \mathrm{~mW} \mathrm{~cm}^{-2}$ ). Cells were irradiated for $0,2.5$, and $5 \mathrm{~min}$ 
and visualized by confocal fluorescence microscopy. Over the time course of irradiation, an increase in the fluorescence intensity was observed for BAD6 (Figure 4). This indicates first
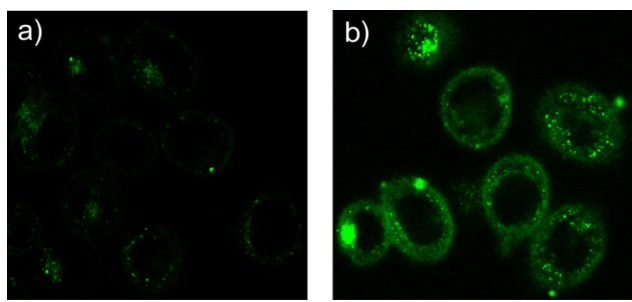

Figure 4. Confocal microscopy images of cells incubated with $1 \mu \mathrm{M}$ of BAD6 after the irradiation with broad-band visible light $(400-700 \mathrm{~nm}$, $23.8 \mathrm{~mW} \mathrm{~cm}^{-2}$ ) for (a) $2.5 \mathrm{~min}$ and (b) $5 \mathrm{~min}$.

that the chromophore entered the cells, rather than simply associating with the external cell membrane; and secondly, that the fluorescence increased in a similar way to that observed for BAD2 in homogeneous solution. However, this behavior was not replicated in the case of BAD5, which showed no observable fluorescence on this time scale, even when irradiated with higher light doses. Lower fluorogenicity of BADS is in accord with the behavior of parent BAD1, which was shown to react with ${ }^{1} \mathrm{O}_{2}$ considerably slower than BAD2.

At higher concentrations of BADs, evidence of morphological changes to the cells upon irradiation, most noticeably "blebbing" of the cell membrane, was observed (Figure S12), indicating apoptotic behavior. Cell viabilities after incubation with a range of concentrations (1-50 $\mu \mathrm{M})$ of BADs 5 and 6 , followed by light treatment $\left(23.8 \mathrm{~mW} \mathrm{~cm}^{-2}\right)$, were assayed by MTT protocol. The results obtained indicate that both water-soluble BADs induce a significant cytotoxic effect on the cells, whereas negligible cytotoxic effects were observed in the control group under otherwise identical conditions, but without irradiation (Figure S13). Median lethal doses $\left(\mathrm{LD}_{50}\right)$ of BADs were found to be 4 $\mu \mathrm{M}$, thus the lower dose of $1 \mu \mathrm{M}$ was selected for imaging experiments.

In conclusion, we have demonstrated that heavy atom-free donor-acceptor dyads can be used as ${ }^{1} \mathrm{O}_{2}$ sensitizers, whereby the triplet excited states form by way of photoinduced electron transfer. Moreover, the described dyads are capable of forming strongly fluorescent species with self-sensitized ${ }^{1} \mathrm{O}_{2}$ in biological media. The fluorescent response allows visualization of ${ }^{1} \mathrm{O}_{2}$ formation within the cells and, consequently, fine-tuning of the photon doses required to cause oxidative stress. These sensitizers may give rise to a promising new class of materials for photonic applications that depend on triplet excited states generation. Studies to extend the scope such systems are underway.

\section{ASSOCIATED CONTENT}

\section{S Supporting Information}

The Supporting Information is available free of charge on the ACS Publications website at DOI: 10.1021/jacs.7b00551.

Experimental details; data for BAD1, BAD1-BE, BAD2$\mathrm{BA}, \mathrm{BAD} 2-\mathrm{TE}$

\section{AUTHOR INFORMATION}

\section{Corresponding Authors}

*filatovm@tcd.ie

*R.W.Boyle@hull.ac.uk

*sengem@tcd.ie
ORCID

Mikhail A. Filatov: 0000-0002-1640-841X

Frédéric Laquai: 0000-0002-5887-6158

Mathias O. Senge: 0000-0002-7467-1654

Notes

The authors declare no competing financial interest.

\section{ACKNOWLEDGMENTS}

This work was supported by grants from the European Commission (M.A.F., CONSORT, Grant No. 655142), Science Foundation Ireland (M.O.S. SFI IvP 13/IA/1894), and by funding from King Abdullah University of Science and Technology (KAUST). We thank Prof. Sergei Vinogradov for helpful discussions.

\section{REFERENCES}

(1) Chan, J.; Dodani, S. C.; Chang, C. J. Nat. Chem. 2012, 4, 973-984.

(2) McGlynn, S. P.; Azumi, T.; Kinoshita, M. Molecular Spectroscopy of the Triplet State; Prentice Hall: New York, 1969; pp 284-325.

(3) (a) Ziessel, R.; Allen, B. D.; Rewinska, D. B.; Harriman, A. Chem. Eur. J. 2009, 15, 7382-7393. (b) Amin, A. N.; El-Khouly, M. E.; Subbaiyan, N. K.; Zandler, M. E.; Fukuzumi, S.; D’Souza, F. Chem. Commun. 2012, 48, 206-208. (c) Huang, L.; Yu, X.; Wu, W.; Zhao, J. Org. Lett. 2012, 14, 2594-2597. (d) Guo, S.; Xu, L.; Xu, K.; Zhao, J.; Kucukoz, B.; Karatay, A.; Yaglioglu, H. G.; Hayvali, M.; Elmali, A. Chem. Sci. 2015, 6, 3724-3737. (e) Cheriya, R. T.; Joy, J.; Alex, A. P.; Shaji, A.; Hariharan, M. J. Phys. Chem. C 2012, 116, 12489-12498. (f) Mani, T.; Niedzwiedzki, D. M.; Vinogradov, S. A. J. Phys. Chem. A 2012, 116, $3598-3610$.

(4) Ragàs, X.; Jiménez-Banzo, A.; Sánchez-García, D.; Batllori, X.; Nonell, S. Chem. Commun. 2009, 2920-2922.

(5) Kamkaew, A.; Lim, S. H.; Lee, H. B.; Kiew, L. V.; Chung, L. Y.; Burgess, K. Chem. Soc. Rev. 2013, 42, 77-88.

(6) Zhao, J.; Xu, K.; Yang, W.; Wang, Z.; Zhong, F. Chem. Soc. Rev. 2015, 44, 8904-8939.

(7) (a) Wan, C. W.; Burghart, A.; Chen, J.; Bergström, F.; Johansson, L. B.; Wolford, M. F.; Kim, T. G.; Topp, M. R.; Hochstrasser, R. M.; Burgess, K. Chem. - Eur. J. 2003, 9, 4430-4441. (b) Benniston, A. C.; Harriman, A.; Whittle, V. L.; Zelzer, M.; Harrington, R. W.; Clegg, W. Photochem. Photobiol. Sci. 2010, 9, 1009-1017.

(8) Sunahara, H.; Urano, Y.; Kojima, H.; Nagano, T. J. Am. Chem. Soc. 2007, 129, 5597-5604.

(9) Aubry, J.-M.; Pierlot, C.; Rigaudy, J.; Schmidt, R. Acc. Chem. Res. 2003, 36, 668-675.

(10) (a) Hattori, S.; Ohkubo, K.; Urano, Y.; Sunahara, H.; Nagano, T.; Wada, Y.; Tkachenko, N. V.; Lemmetyinen, H.; Fukuzumi, S. J. Phys. Chem. B 2005, 109, 15368-15375. (b) Lifschitz, A. M.; Young, R. M.; Mendez-Arroyo, J.; Roznyatovskiy, V. V.; McGuirk, C. M.; Wasielewski, M. R.; Mirkin, C. A. Chem. Commun. 2014, 50, 6850-6852.

(11) Kotani, H.; Ohkubo, K.; Fukuzumi, S. J. Am. Chem. Soc. 2004, 126, 15999-16006.

(12) Levanon, H.; Norris, J. R. Chem. Rev. 1978, 78, 185-198.

(13) Dance, Z. E. X.; Mi, Q.; McCamant, D. W.; Ahrens, M. J.; Ratner, M. A.; Wasielewski, M. R. J. Phys. Chem. B 2006, 110, 25163-25173. (b) Dance, Z. E. X.; Mickley, S. M.; Wilson, T. M.; Ricks, A. B.; Scott, A. M.; Ratner, M. A.; Wasielewski, M. R. J. Phys. Chem. A 2008, 112, 41944201. (c) Colvin, M. T.; Ricks, A. B.; Scott, A. M.; Co, D. T.; Wasielewski, M. R. J. Phys. Chem. A 2012, 116, 1923-1930.

(14) Maus, M.; Rettig, W.; Bonafoux, D.; Lapouyade, R. J. Phys. Chem. A 1999, 103, 3388-3401.

(15) (a) Rigaudy, J.; Baranne-Lafont, J.; Defoin, A.; Kim Cuong, N. Tetrahedron 1978, 34, 73-82. (b) Rigaudy, J.; Scribe, P.; Breliere, C. Tetrahedron 1981, 37, 2585-2593.

(16) (a) Zehm, D.; Fudickar, W.; Hans, M.; Schilde, U.; Kelling, A.; Linker, T. Chem. - Eur. J. 2008, 14, 11429-11441. (b) Baral, R. N.; Thomas, S. W. J. Org. Chem. 2015, 80, 11086-11091. 